Helgoländer wiss. Meeresunters. 17, 435-444 (1968)

\title{
Vergleichende Untersuchungen über Hydrochemie und Plankton deutscher Flußmündungen
}

\author{
HeinRICH KüHL und Hans ManN \\ Bundesforschungsanstalt für Fischerei, Institut für Küsten- und Binnenfischerei, \\ Hamburg 50
}

\begin{abstract}
Comparative investigations on hydro-chemistry and plankton of German estuaries. The three German rivers Elbe, Weser and Ems were examined in regard to their chemical mechanisms and their phyto- and zooplankton distributions. The subdivision of the examined river sections into salinity zones has proved to be satisfactory. Their expansion is determined by the topography as well as by the amount of discharge of the river. Increase and decrease of plankton communities of fresh- and salt-water are based thereon, whereby the supply of sewage water of big towns is of great importance. The lower regions of the examined rivers have their own endemic communities. Many species exhibit two maxima, one within the limnic region, the other in the polyhalinicum. Knowledge of the present plankton communities is important in order to be able to recognize any changes which may be caused through increased industrial drainages.
\end{abstract}

\section{EINLEITUNG}

In den Mündungsgebieten von Tideflüssen treffen das Süßwasser des Flusses und das Seewasser, das bei Flut in die Flußmündung eindringt, aufeinander und mischen sich in breiter Front. Hierdurch werden nicht nur der Verlauf der Strömung, die Ausbildung der hygrographischen Faktoren und die Zusammensetzung der Organismenwelt in großem Umfang bestimmt, sondern dieses Geschehen muß auch bei der Einleitung von $\mathrm{Abwässern} \mathrm{in} \mathrm{die} \mathrm{Flußmündungen} \mathrm{oder} \mathrm{flußnahe} \mathrm{Küstengebiete} \mathrm{berück-}$ sichtigt werden. In der Mischungszone von Fluß- und Seewasser überlagern sich Dichteund Tideströme, wodurch es zu sehr schwer übersehbaren Schichtungsverhältnissen kommen kann. In einem tidefreien Flußabschnitt lassen sich aus der Menge des eingeleiteten Abwassers und der Wasserführung des Flusses die Verdünnungsmöglichkeiten und damit auch die Höhe der Abwasserbelastung berechnen. Ganz anders liegen die Verhältnisse in einem von den Tiden beeinflußten Gebiet, wo das Wasser viermal am Tage seine Abflußrichtung ändert. Die Dauer einer Tide (Ebbe und Flut) ist durch astronomische Gegebenheiten festgelegt, doch werden Steig- und Falldauer sehr stark von der Windrichtung und der Windstärke beeinflußt; außerdem spielt die mit dem Fluß herangeführte Menge an Oberwasser eine große Rolle für die Fortschrittszeiten und Fortschrittsgeschwindigkeiten der Tidewelle. Mit zunehmendem Oberwasser wird 
der Ebbstrom größer und der Flutstrom kleiner (HENSEN 1953). Da mit wachsendem Oberwasser auch größere Wassermengen herangeführt werden, tritt eine Hemmung der Flutwelle ein, deren Bewegung also zeitlich verzögert wird. Außerdem wirken sich natürlich auch wasserbauliche Veränderungen, wie z. B. Vertiefung der Flußsohle oder Einbauten von Buhnen oder Leitdämmen auf den Ablauf der Tidebewegung aus. So hat man z. B. festgestellt, daß auf der Unterweser das Tidehochwasser nach dem Ausbau auf der Strecke Bremerhaven-Brake langsamer aufläuft als vor dem Ausbau.

\section{ERGEBNISSE UND DISKUSSION}

Die hydrographischen Verhältnisse wirken sich auch auf die Hydrochemie der Flußmündungen aus. In Untersuchungen an den Mündungen der großen deutschen Flüsse Elbe, Weser, Ems (Abb. 1) konnten wir feststellen, daß als wesentlicher Faktor für die Ausbildung des hydrochemischen Geschehens das Zusammentreffen von Süßund Salzwasser anzusehen ist. Je nach dem Grad der Durchmischung bilden sich

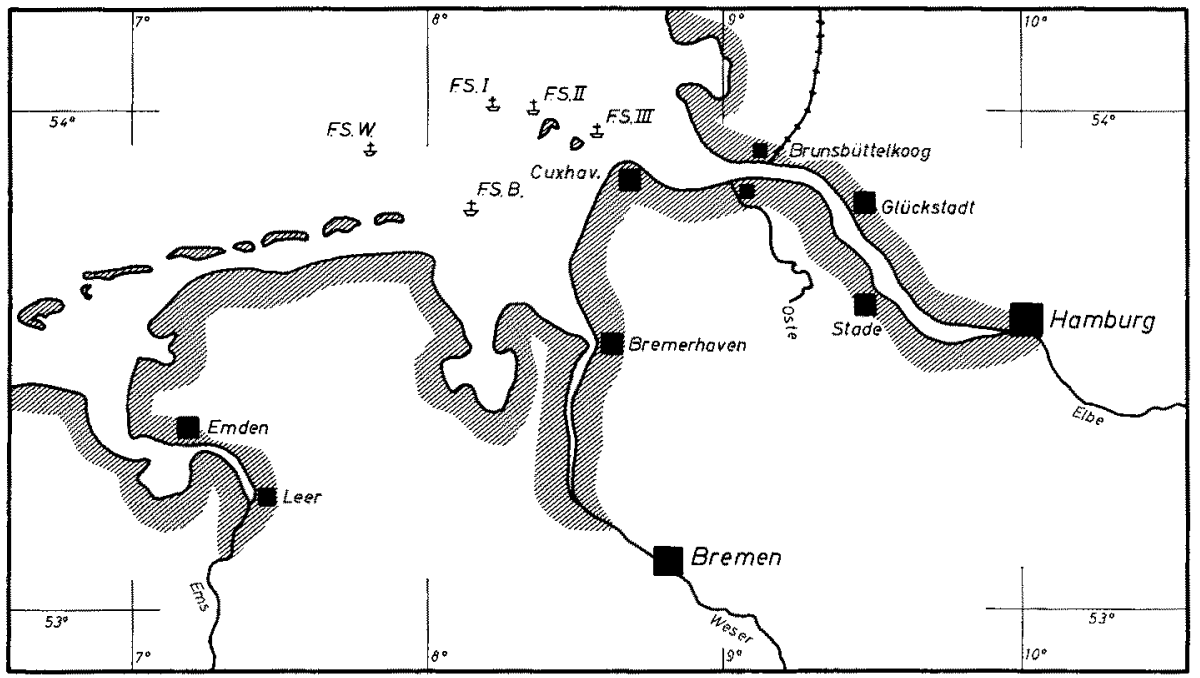

Abb. 1: Ubersichtsbild für die drei untersuchten Flußmündungsgebiete: Elbe von Hamburg bis FS Elbe 1, Weser von Bremen bis Weser FS, Ems von Leerort bis Hubertgat

Zonen unterschiedlichen Salzgehaltes aus. Da der Seewasserkörper durch die Gezeitenbewegungen mehr oder weniger weit in den Mündungstrichter hineingedrückt wird, wandern die Brackwasserzonen dementsprechend weit flußauf- und flußabwärts. Diese Veränderungen, die naturgemäß durch Wetterverhältnisse (Wind) in ihrem Ausmaß beeinflußt werden können, sind nur von kürzerer Dauer entsprechend dem Tideverlauf. Langfristige Verschiebungen kommen im Zusammenhang mit Schwankungen der Oberwassermenge zustande, da sie das gesamte Mischungsgebiet flußabwärts oder -aufwärts verlagern. Die Schwankungen in der Oberwassermenge wirken sich daher auch biologisch stärker aus als solche der Gezeiten. 
Die Menge des Süßwassers, d. h. die durchschnittliche Oberwasserführung und die Morphologie des Mündungstrichters, spielt eine wesentliche Rolle für die Ausbildung der Brackwasserzone (Abb. 2). So sind diese Zonen z. B. bei der Elbe wesentlich ausgedehnter als in der Ems. Trotz der Verschiedenheiten in der Morphologie und Hydrologie zeigen die von uns untersuchten Flüsse gewisse Regelmäßigkeiten, die sich wiederholen. Abweichungen erklären sich durch die besondere Gestalt der Flüsse. Neben diese naturgegebenen Komponenten treten die Bedingungen, die durch den Menschen künstlich geschaffen wurden. Es sind dies die oben bereits genannten wasserbaulichen Veränderungen; von großer Bedeutung sind aber auch die menschlichen Ansiedlungen. Sie bringen häusliche und industrielle Abwässer, die durch ihren biologischen Abbau den Chemismus des Tideflusses wesentlich beeinträchtigen. Am wenigsten wird davon der Verlauf der Salzgehaltskurven beeinflußt. Im oberen Bereich der Mischungszone beginnt der Salzgehalt langsam und gleichmäßig anzusteigen, dann folgt eine Region, in der der Salzgehalt sprunghaft zunimmt (Sprungzone). Danach gleicht er sich wieder langsam dem Salzgehalt des Westwassers (euhaline Zone) an.

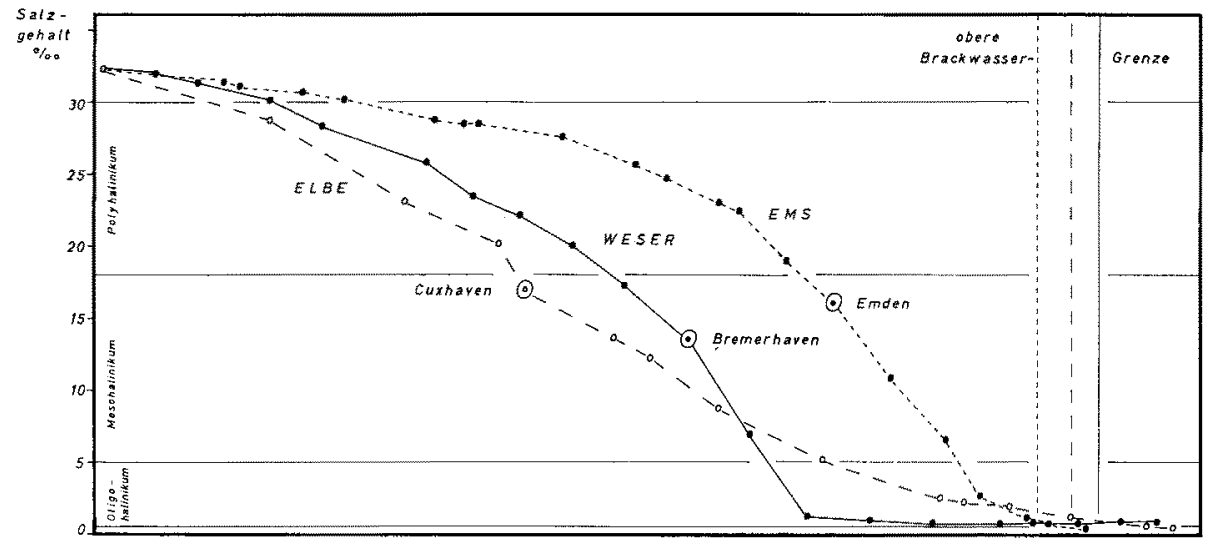

Abb. 2: Durchschnittssalzgehalt, errechnet aus allen durchgeführten Untersuchungsfahrten bei Ebbe und Flut. Wasserführung der Flüsse an den Untersuchungstagen. Elbe: $220-1150 \mathrm{~m}^{3} / \mathrm{sec}$ an Pegel Darchau; Weser: $140-380 \mathrm{~m}^{3} / \mathrm{sec}$ an Pegel Inschede; Ems: $15-73 \mathrm{~m}^{3} / \mathrm{sec}$ an Pegel Herbrum

Die größten Schwankungen treten in der Sprungzone auf, hier findet man auch die stärksten Unterschiede zwischen Oberfläche und Tiefe. Dieses Gebiet liegt für die Elbe in der pleio-mesohalinen und polyhalinen Zone (vergl. Abb. 3 bis 5), entsprechend der geringeren Wasserführung der Weser ist die Sprungzone dieses Gebietes weiter flußaufwärts in die mesohaline und bei der Ems in die meso- und oligohaline Zone verschoben. Bei der Ems macht sich also bemerkbar, daß ein kleiner Süßwasserkörper auf den verhältnismäßig großen Dollart mit Seewasser stößt (KüHL \& MANN 1953, 1954, 1957).

Charakteristisch für die Tideflüsse ist neben dem Salzgehalt die Ausbildung von Trübungszonen. Mit steigendem Salzgehalt nimmt der Grad der Trübung zu und erreicht in der mesohalinen Zone ihren Höhepunkt. Danach geht sie wieder zurück, 
und das Wasser wird nach See hin deutlich klarer. Ein kleineres, zweites Trübungsmaximum kann sich an der Grenze der meso- zur polyhalinen Zone ausbilden, wie dies z. B. in der Außenelbe der Fall ist. Die Trübungszonen, in denen die Trübungen in Form von Wolken auftreten, haben eine Ausdehnung von 20 bis $30 \mathrm{~km}$. Ahnlich den Salzgehaltszonen werden sie mit den Tiden flußauf- und -abwärts verschoben. Die

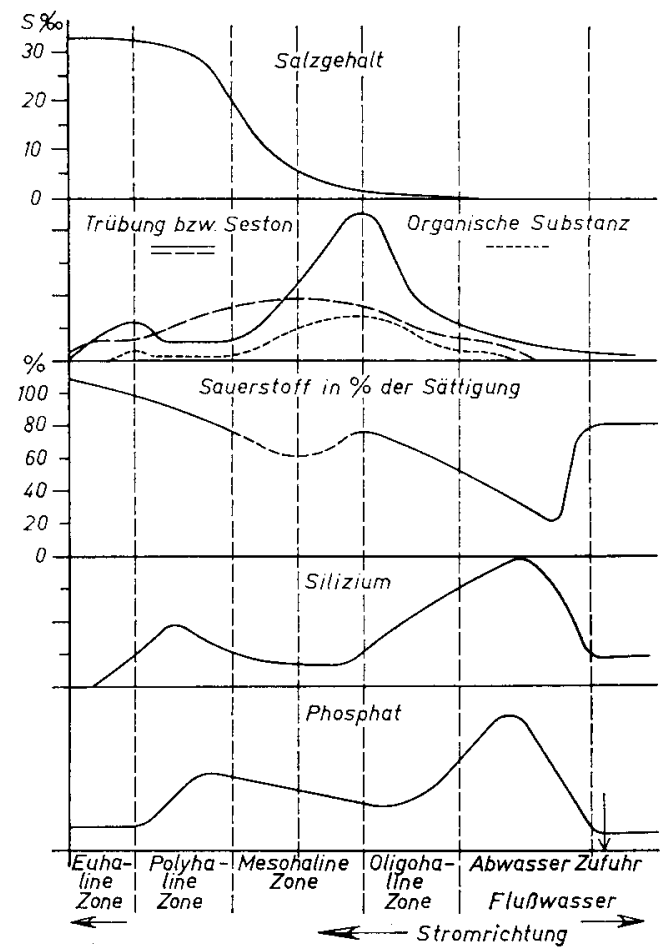

Abb. 3: Salzgehalt, Trübung, Sauerstoffsättigung und Gehalt an Silizium und Phosphat in einer Flußmündung, schematisiert nach Untersuchungen in Elbe, Weser und Ems.

(Nach KüHL \& MANN 1961)

Oberwassermenge bestimmt die Form der Trübungszone. Bei großer Wasserführung ist die Trübungskurve lang und niedrig, bei geringem Süßwasserzufluß kurz und hoch (Kühl \& ManN 1953, 1954, 1957, Lüneburg 1950/1951, Mann 1958).

Die bisher besprochenen Faktoren tragen natürlichen Charakter, das gilt nicht in gleichem Maße für den Sauerstoffgehalt des Wassers. Normalerweise hat das Flußwasser eine Sauerstoffsättigung von 80 bis $90 \%$. Unterhalb von Abwassereinleitungen sinkt der Gehalt rapide $a b$, die Höhe und Dauer des Absinkens hängen von der Stärke der Belastung mit fäulnisfähigen sauerstoffzehrenden Stoffen, der Wassertemperatur und dem Grad der Verdünnung durch das Flußwasser ab. Im Zusammenhang mit der Belastung kommt es zu einer mehr oder weniger langen Selbstreinigungszone, an deren Ende der Sauerstoffgehalt wieder auf 80 bis $90 \%$ ansteigt (Abb. 3). Innerhalb der Sprungzone können örtliche Störungen als Folge von Abbauprozessen auftreten. 
Wesentlich ist, daß die Löslichkeit des Sauerstoffs im Meerwasser bei gleicher Temperatur um etwa $1 / 5$ niedriger ist als im Süßwasser. Diese Tatsache muß bei der Einleitung von organischen Substanzen in Küstengewässer stets berücksichtigt werden.

Im Zusammenhang mit dem Abbau der dem Fluß zugeführten häuslichen Abwässer ist das Verhalten der anorganischen Stickstoffverbindungen von besonderem Interesse.

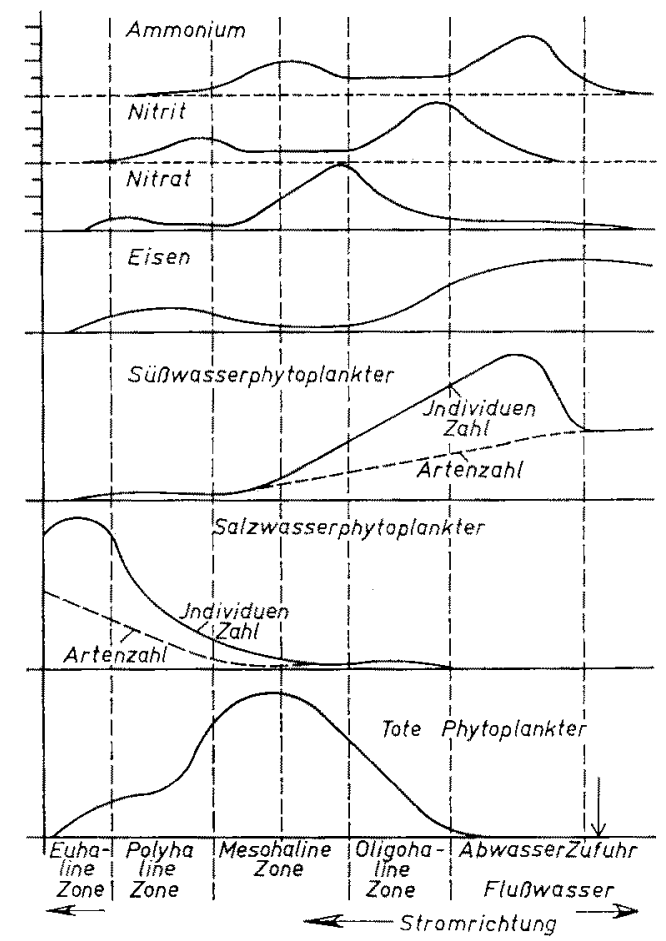

Abb. 4: Gehalt an Ammonium, Nitrit, Nitrat und Eisen sowie die Verbreitung von Süß- und Salzwasserphytoplanktern in einer Flußmündung, schematisiert nach Untersuchungen an Elbe, Weser, Ems. (Nach KüHL \& MaNn 1961)

Bei dem natürlichen Abbau der Eiweißstoffe entsteht als anorganisches Endprodukt Ammoniak, das dann in weiterem Verlauf zu Nitrit und Nitrat mineralisiert wird. Diese Prozesse sind in stehenden Gewässern leichter zu verfolgen als in fließenden, da hier sich oft die Turbulenz störend bemerkbar macht. Doch ging in allen Flüssen grundsätzlich der Ablauf in gleicher Weise vor sich. Es sei darauf hingewiesen, daß die Nitratwerte stets erst dann anstiegen, wenn die Ammoniakwerte schon ihren Höhepunkt überwunden hatten (Abb. 4).

Von großem Interesse war nun, die Beziehungen zwischen den hydrochemischen Faktoren und der Ausbildung der Planktongemeinschaften zu untersuchen. Der Fluß bringt zunächst eine bestimmte Planktongemeinschaft mit sich, die naturgemäß aus Süßwasserformen besteht. Triff diese Lebensgemeinschaft in der oligohalinen Zone auf den erhöhten Salzgehalt, so stirbt eine Anzahl von Formen ab, die diese Milieu- 
veränderung nicht vertragen. Die Zahl dieser Arten nimmt mit steigendem Salzgehalt schnell zu. Nur einige halophile Formen überstehen eine größere Versalzung des Wassers. Parallel zur weiteren Zunahme des Salzgehalts wächst jetzt die Zahl der Salzwasserformen, die ihr Maximum in der polyhalinen Zone finden. Betrachtet man die Veränderungen in den Planktongemeinschaften von See her, so zeichnet sich dort das gleiche Geschehen wie bei den Süßwasserformen ab. Mit abnehmendem Salzgehalt geraten die Salzwasserformen ins Pessimum und sterben $a b$. In Gegensatz zu den Süßwasserformen geht aber der Abbau der marinen Phytoplanktongemeinschaft sehr viel schneller vor sich (Abb. 4). Die meisten marinen Formen dringen nicht über die 8-0/00-Grenze weiter ins Süßwasser vor.

Die drei untersuchten Flüsse (Elbe, Weser, Ems) unterscheiden sich nur geringfügig durch ihr Artenspektrum, doch hängt die Form der Verteilung von der Ausdehnung der halinen Zonen $a b$, die wiederum von dem Mischungsverhältnis des Süß- und Seewassers bestimmt wird. Im allgemeinen wurden von allen von uns untersuchten Gruppen des Zooplanktons zwei Maxima ausgebildet. Das eine lag im limnischen Bereich und erstreckte sich bis in die oligohaline Zone, das zweite in der polyhalinen Zone. Bei den Würmern wird das Maximum im limnischen Bereich durch Oligochaeten gestellt, im marinen Bereich durch Polychaetenlarven. Unter den Crustaceen bilden im Süßwasser die Daphniden und Bosminen, unter den Copepoden besonders Eurytemora affinis ein Maximum, während im Brackwasser die Cladoceren eine untergeordnete Rolle spielen, dagegen die Copepoden durch mehrere Arten vertreten sind. Die Mysideen findet man noch ziemlich weit in einzelnen Arten (Mesopodopsis slabberi und Neomysis integer) flußaufwärts, doch haben sie ihre maximale Entwicklung in der poly-und mesohalinen Zone.

Zooplankter marinen Ursprungs haben naturgemäß ihr stärkstes Auftreten in der polyhalinen Zone, sie dringen unter normalen Verhältnissen nicht über die meiomesohaline Zone flußaufwärts. Wenige euryplastische Formen, wie z. B. Eurytemora affinis kommen im gesamten Flußabschnitt der Elbe vor.

Ahnlich wie bei dem Phytoplankton kommt es in dem Gebiet zwischen den beiden Zonen maximaler Entfaltung zu einem Bereich mit minimaler Zooplankton-Entwicklung. Durch die Absterbevorgänge in diesem Bereich werden viele Nährstoffe frei, die den Planktern im seewärts liegenden Abschnitt zum Aufbau dienen, wie dies für die Stickstoffverbindungen, das Phosphat und Silizium auf Abbildung 3 und 4 gezeigt ist. Wie oben bereits dargestellt, können die Bereiche der maximalen Planktonentwicklung durch besondere Wetterverhältnisse oder außergewöhnliche Wasserführung der Flüsse verschoben werden.

Deutlichen Einfluß auf die Entwicklung der Planktongemeinschaften haben die häuslichen Abwässer, die unterhalb der Städte z. B. Hamburg, Bremen, Emden eingeleitet werden. Die mit den Abwässern dem Fluß zugeführten organischen Stoffe dienen den Phytoplanktonorganismen als Nährstoffe. Als Folge kommt es unterhalb dieser Städte zu einer Planktonblüte, auf der sich wiederum eine Zooplanktongemeinschaft aufbaut. Besonders beachtenswert ist es, daß in den drei untersuchten Flußmündungen, im ganzen gesehen, die gleichen Arten angetroffen werden. Als wichtiges Ergebnis unserer Untersuchungen ist anzusehen, daß die Flußmïndungen charakteristische endemische Lebensgemeinschaften besitzen. Daneben gibt es natürlich auch eine 
Anzahl Arten, die als Irrgäste anzusehen sind, die durch besondere hydrographische Verhältnisse in die Flußmündung hin verfrachtet werden.

Altere Untersuchungen über das Flußplankton der unteren Regionen des Brackwasserbereichs gibt es kaum; unsere Kenntnisse sind erst in der letzten Zeit erweitert worden. Uber die Außenelbe lagen bei Beginn unserer Untersuchungen nur die Arbeiten von Kirchenpauer (1862), Daml (1892) und Thimemani (1934) vor, der limnische Bereich der Unterelbe war dagegen besser bekannt. Uber die Weser liegen von SChrÄDER (1941) einige Angaben über das Plankton vor. Über das Plankton der Ems sind uns keine Arbeiten bekannt.

Der Grund, warum das Plankton der Flußmündungen früher so wenig untersucht wurde, ist darin zu suchen, daß die Untersucher des Küstenplanktons annahmen, daß die mit dem Küstenstrom von Westen herangeführten marinen Plankter mit den Tideströmungen in den Fluß verdriftet werden, um im Brackwassergebiet bei vermindertem Salzgehalt abzusterben. Sofern es sich um euryhaline Formen handelte, könnten sie noch eine Zeitlang am Leben bleiben. Es würde sich also um eine „sterile Zerstreuung“ handeln. Die Forschungsschiffe der früheren Untersuchungen blieben dem „schmutzigen Flußwasser" möglichst fern.

Durch unsere Untersuchungen des Planktons im Unterlauf der deutschen Flüsse konnte aber gezeigt werden, daß eine nicht geringe Zahl von marinen Arten nicht nur in die Flußmündungen eindringen und den verminderten Salzgehalt gut vertragen kann. Viele Arten pflanzen sich auch im Polyhalinikum fort, so daß es sich hier also um echte Endemismen handelt. Es gibt sogar einige Arten, die im nährstoffreichen Mündungsgebiet Massenentwicklungen aufweisen.

Besonders auffallend ist das Auftreten der Medusen in den Ästuaren, da gerade sie von den Strömungen sehr leicht verfrachtet werden können. Hierüber gibt es fast keine älteren Angaben. Es konnte der Nachweis erbracht werden, daß für eine Anzahl von Arten die gesamte Entwicklung im Polyhalinikum verläuft. Dies gilt z. B. für die Hydromedusen Sarsia tubulosa, Ratbkea octopunctata, Margelopsis baeckeli, Eucheilota maculata und Obelia spsp. Von Ratbkea octopunctata konnte bei Cuxhaven der Polyp zum ersten Male im Freien gefunden werden, nachdem er bisher nur aus der Züchtung bekannt war (KüHL 1962, 1964, 1967). Seit dem Jahre 1949 ist die Margelide Nemopsis bachei AGAssiz, die früher in der Zuidersee vorkam und nach der Abschleusung verschwand, in der Elbemïndung aufgetreten. Sie ist seitdem eine Massenform des Polyhalinikum. Es handelt sich um eine Brackwasserform, die von uns aber bisher in der Ems noch nicht gefunden wurde.

Der zierliche schwimmende Polyp Clytia pelagica (VAN BENEDEN) konnte von uns häufig in allen drei untersuchten Flußmündungen gefunden werden, und zwar in allen Graden der Verzweigung, daß es zweifelhaft ist, ob er wirklich nur als „Leitform des vor der belgisch-holländischen Küste befindlichen flämischen Küstenwassers" zu gelten hat (KünNE 1937, 1952). Es kommt noch hinzu, daß das Schlüpfen der phialidium-ähnlichen Meduse im Elbeästuar beobachtet werden konnte. KüNNE (1952) selbst schließt jedoch die Möglichkeit nicht aus, daß „Clytia pelagica doch irgendwo an der Küste der südöstlichen Nordsee, vielleicht im Wattenmeer, dauernd vorhanden ist, aber bisher übersehen worden ist". Das gleiche gilt für die Scyphomeduse Rhizostoma octopus, die in der Elbmündung regelmäßig jedes Jahr in der Zeit von Juni bis 

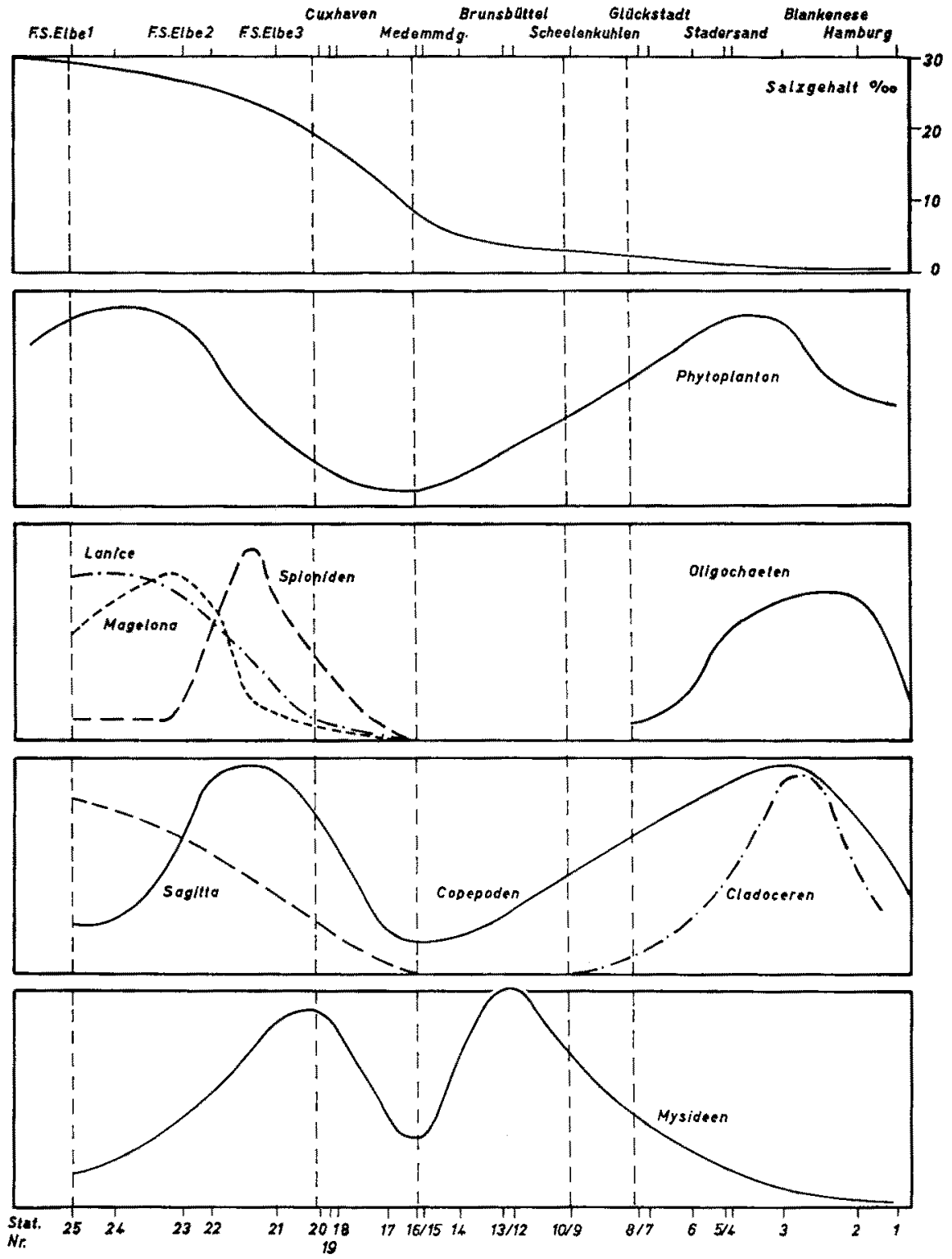

Abb. 5: Salzgehalt und Maxima des Phyto- und Zooplanktons in einer Flußmündung. Schematische Darstellung nach Untersuchungen an der Elbe. (Nach KüHL \& ManN 1962 und 1967)

Oktober auftritt und deren ganze Entwicklung in der Elbmündung verfolgt werden kann (KüHL 1964, 1967, ThIEL 1966). Es ist nicht auszuschließen, daß auch die Kompaßqualle Chrysaora byoscella und die blaue Nesselqualle Cyanea lamarckii in 
den Flußmündungen heimisch sind, wohingegen dies für die gelbe Nesselqualle Cyanea capillata und die euryhaline Aurelia aurita nicht im gleichen Maße gilt.

Auch die Ctenophoren Pleurobracbia pileus und Beroe cucumis durchlaufen ihre gesamte Entwicklung im Polyhalinikum der Flußmündungen. Sie erreichen aber nicht die gleiche Größe wie die Nordseetiere.

Zum Astuarplankton gehören naturgemäß die Larven der wattbewohnenden Würmer, wie Spioniden, Terebelliden, Polynoiden, Nereiden u. a., die Larven von Cirripediern, Carcinus und Crangon, ferner die von Ophiuren und irregulären Seeigeln (Echinocyanus und Echinocardium), Bivalven- und Gastropodenlarven (Mytilus, Mya, Macoma, Litorina u. a.). Schließlich sind noch die Chätognaten Sagitta setosa und von den Copelaten Oikopleura dioica, bedingt auch Fritillaria (Ems) zu nennen.

Die Untersuchungen zeigten also deutlich, daß die Flußmündungen eine eigene Planktongesellschaft besitzen. Abbildung 5 stellt die Zusammenhänge zwischen Salzgehalt, Verteilung des Phytoplanktons und einiger Vertreter des Zooplanktons dar. Die Kenntnis dieser Planktongesellschaften, ihre Zusammensetzung, jahreszeitliche Veränderung, besonders auch die Abhängigkeit von der Abflußmenge der Flüsse ist wichtig für die Beurteilung von Abwasserschäden im Tidegebiet, in mancher Beziehung ist sie auch methodisch einfacher und geeigneter als eine Beurteilung durch Benthosorganismen.

\section{ZUSAMMENFASSUNG}

1. Die drei großen deutschen Flüsse Elbe, Weser und Ems wurden von uns in mehreren Fahrten auf ihren Chemismus und die Verteilung des Phyto- und Zooplanktons hin untersucht.

2. Die Unterteilung der untersuchten Flußabschnitte in haline Zonen hat sich gut bewährt. Ihre Ausdehnung wird durch die Topographie und die Abflußmenge des Flusses bestimmt. Hierauf gründet sich Auf- und Abbau der Planktongemeinschaften des Süß- und Salzwassers, wobei die Zufuhr von Abwässern großer Städte von Bedeutung ist.

3. Die Unterläufe der untersuchten Flüsse haben eigene endemische Lebensgemeinschaften. Es bilden sich für viele Arten zwei Maxima aus, von denen das eine im limnischen Bereich, das andere im Polyhalinikum liegt.

4. Die Kenntnis der jetzigen Planktongemeinschaften ist wichtig, um Veränderungen durch Einleitungen von industriellen Abwässern zu erkennen.

\section{ZITIERTE LITERATUR}

Bursche, E. M., Kühl, H. \& ManN, H., 1958. Hydrochemie und Phytoplankton in der Unterelbe. Veröff. Inst. Meeresforsch. Bremerh. 5, 165-192.

- - 1959. Beziehungen zwischen dem Chemismus und der Phytoplanktonentwicklung auf der unteren Weser. Int. Revue ges. Hydrobiol. 44, 277-298.

DAHL, F. R., 1892. Untersuchungen über die Tierwelt der Unterelbe. Ber. Commn wiss. Unters. dt. Meere 6 (17-21), 149-185. 
Hensen, W., 1953. Das Eindringen von Salzwasser in die Gezeitenflüsse, in Seekanäle und in Häfen. Mitt. hannover. VersAnst. Grundb. Wassban 3, 20-49.

Kirchenpauer, G. H., 1862. Die Seetonnen der Elbmündung. Abh. Geb. Naturw. Hamb. 4 (2), $1-59$.

KüHL, H., 1962. Die Hydromedusen der Elbmündung. Abb. Verb. naturw. Ver. Hamb. 6, 209-232.

- 1964a. Die Mysideen der Elbmündung, Abh. Verb. naturw. Ver. Hamb. 8, 167-178.

- 1964b. Die Scyphomedusen der Elbmündung. Veröff. Inst. Meeresforsch. Bremerh. 9, 84-94.

- 1967. Die Hydromedusen der Emsmündung. Veröff. Inst. Meeresforsch. Bremerb. 10, 239 bis 246 .

- \& MANn, H., 1953. Beiträge zur Hydrochemie der Unterelbe. Veröff. Inst. Meeresforsch. Bremerb. 2, 236-268.

- - 1954. Beiträge zur Hydrochemie der Ems. Veröff. Inst. Meeresforsch. Bremerh. 3, 126 bis 158 .

- - 1957. Beiträge zur Hydrochemie der Weser. Veröff. Inst. Meeresforsch. Bremerh. 5, $34-62$.

- - 1958. Das Verhalten anorganischer Stickstoffverbindungen im Mündungsgebiet eines Flusses. Arch. FischWiss. 9, 9-16.

- - 1961. Vergleichende hydrochemische Untersuchungen an den Mündungen deutscher Flüsse. Verh. int. Verein. theor. angew. Limnol. 14, 451-458.

- - 1962. Über das Zooplankton der Unterelbe. Veröff. Inst. Meeresforsch. Bremerh. 8, $53-70$.

- - 1967. Untersuchungen tiber das Plankton der Außenelbe. Gewäss. Abreäss. (im Druck).

KüNNE, C., 1937. Uber die Verbreitung der Leitformen des Großplanktons in der südlichen Nordsee im Winter. Ber. dt. wiss. Kommn Meeresforsch. 8, 131-164.

- 1952. Untersuchungen über das Großplankton in der Deutschen Bucht und im Nordsylter Wattenmeer. Helgoländer wiss. Meeresunters. 4, 1-54.

LüNeBurg, H., 1950/51. Über Messung und Bedeutung der Sinkstoffe in Elbe und Weser. Vom Wass, 18, 197-207.

ManN, H., 1958. Abwasserprobleme in Tideflïssen. Ber. abwassertech. Verein. 9, 101-112.

SChräder, T., 1941. Hydrographie, Biologie und Fischerei der Unter- und Außenweser. (Fischereibiologische Untersuchungen im Wesergebiet. II.) Z. Fisch. 39, 527-693.

Thiemann, K., 1934. Das Plankton der Flußmündungen. Wiss. Ergebn. dt. atlant. Exped. ,Meteor $12(1), 199-273$. 